Website: http://jsci.utq.edu.iq Email: utjsci@utq.edu.iq إنتشار طفيلي Fasciola gigantica في المضيف الوسطي في محافظة النجف الاشرف

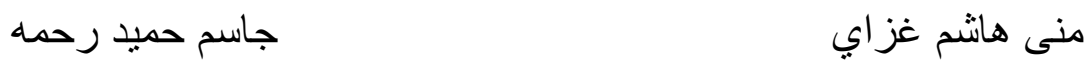

الخلاصة:

شملت الدراسة الحالية التحري عن نسب الاصـابة بطفيلي ديدان الكبد العملاقة Fasciola gigantica في المضائف الوسطية في محافظة النجف في الفترة من تموز 2009 لغاية حزيران 2010 فحصت عينات المضائف الوسطية Lymneae auricularia التي جمعت

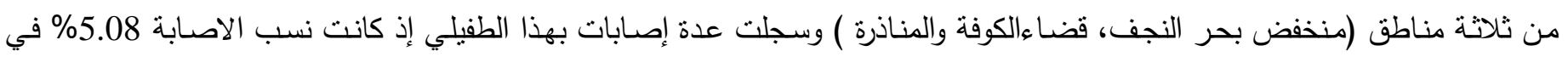
المناذرة ،2.45 \% في الكوفة و0.92\% في منخفض بحر النجف.

الوسط يساعد على عملية فقس البيوض ، وتحرر المهدبات بفترة

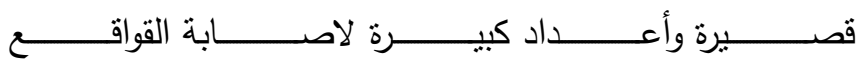
( الطيف والصقر , 1988) ثالثاًّ. الحرارة: إن معدل درجة الحرارة في الليل والنهار الملائهة

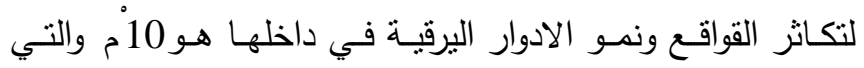

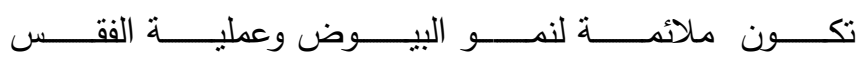

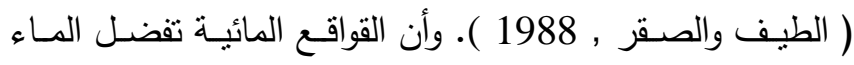
الضحل ودرجات الحرارة التي لاتزيد عن 25 -30مْوالتي نجدها

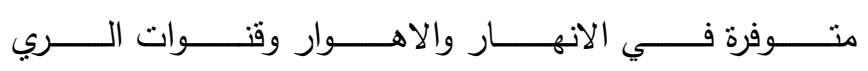

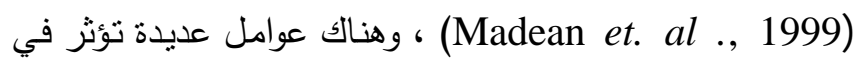

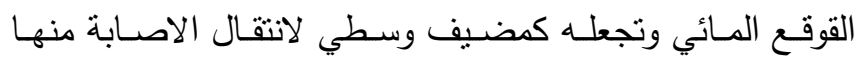

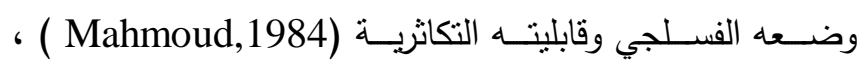
أكدت (1998) Farage أن لاعداد المضيف الوسطي أثراً في

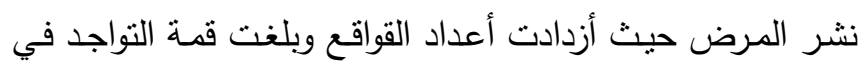

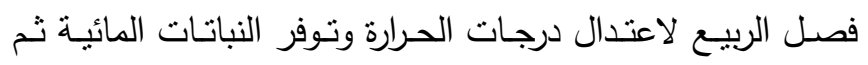

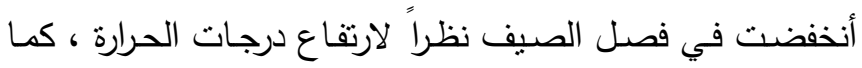
ذكرت أن نسب الاصابة بين القواقع تراوحت بين 10 -40 \% وأن أعلى نسبة أصـابة سجلت في الصبف وقد يكون هذا سبباً في انخفاض كثافة القواقع المائية في هذا الفصل .
المقدمة: بنئ Wilson \& Taylor (1978) أن نسبة انتشار دودة

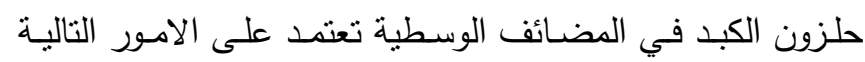
منها: أحتماليـة وصـول البيضـة الى البيئة المناسبة التي تتواجد

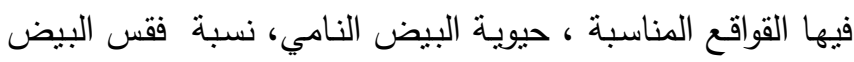
، احتمالية اختراق المهدبة للقوقع وتحقيقها لاصابة ناجحة ـ ترنبط وبائية المرض بالعوامل التي تتحكم بحركة وتكاثر القواقع الناقلة

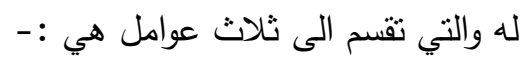

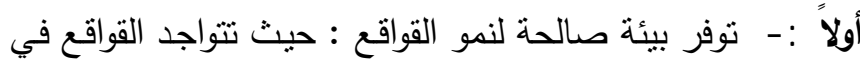

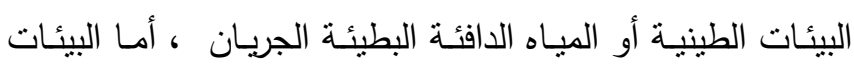

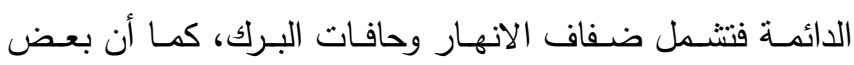
الامـاكن تكون ( بيئات مؤقتة ) خاصـة بعد هطول كميات عالية من الامطار وأثناء الفيضانات إذ تتجمع المياه في الحفر والطيات الارضية ( الطيف والصقر , 1988 ) . ثانياً:. الرطوبة:إذ تعد الرطوبة عاملاً حيوياً جيداً للقواقع التي تعد مضيف وسطي لطفيلي F.gigantica ويعد الجفاف من العوامل القاتلــة للقواقـع وأن الرطوبـة المتلىى لتكـاثز القواقـع ونمـو الادوار اليرقيـة داخل القوقـع هي عندما تكون معدلات المطر أو الميـاه

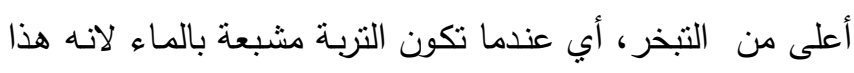


ايران فإن نسبة الاصـابة في القوقع Lendrosiana بطفيلي

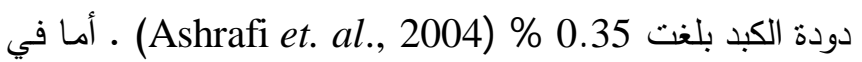

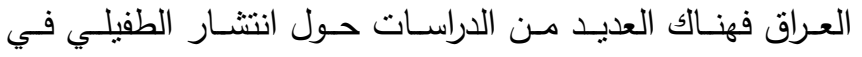
المضائف الوسطية، إذ ذكر بأن معدل أصابة القواقع في العراق

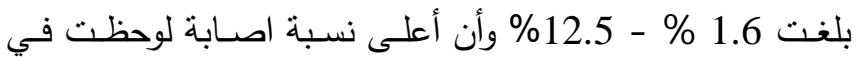

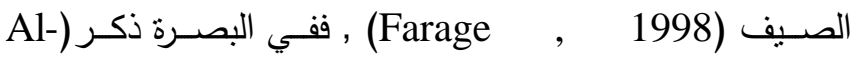
(Mayah,2004 بأن معدل الاصـابة قد بــ 6.3 \% و 7.1\% 7.1 \%

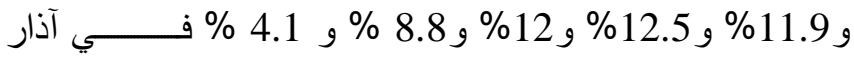
ونيسان وآيار وحزيران وتموز وآب وأيلول على النوالي وذلك في ولاتل قرية الدوة، أما بقية الاثشهر فلم تلاحظ خلالها أصابات ، أما في وابي

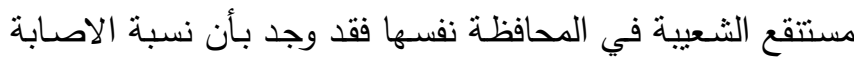
في القواقع كانت 4.6 \% و 9.6 \% و 4.2 \% و 1.6 1. أشهر حزيران وتموز وآب وأيلول على التوالي أما بقية الاشهر فلم

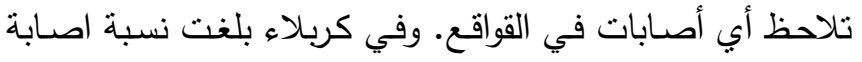
المضـائف الوسطية (القواقع) L.auricularia بيرقـات طفيلي F.gigantica

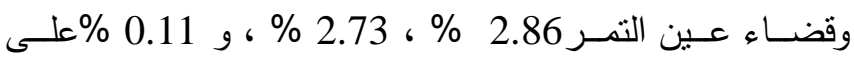
التوالي( الجبوري ،2008 )،و بين الاسدي، (2007) في العراق أن القوقع L.auricularia هو المضيف الوسطي الوحيد لايدان F.gigantica

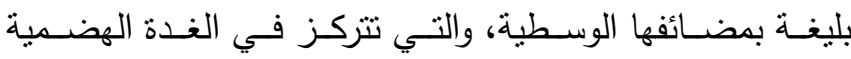

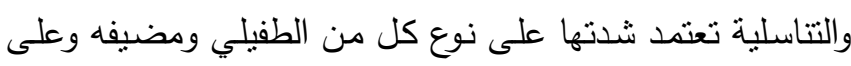
عمر القوقع ومدة التعرض للاصابة وحالة المضيف ودرجة الحرارة

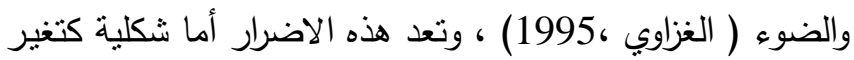

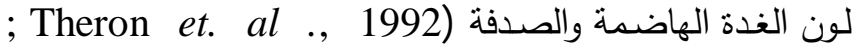
الغزاوي ،1995) أو آلية كالضغط الذي تسلطه الاطوار اليرقية

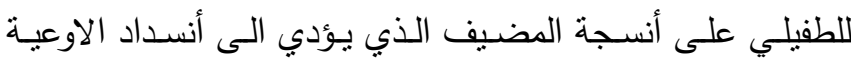

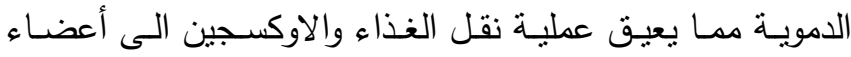
الجسم المختلفة (Thompson,1997) أو فسلجية كتأثيرها على الأى العيد

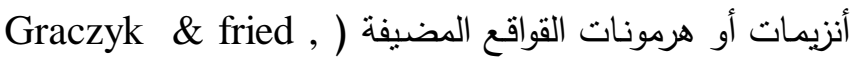

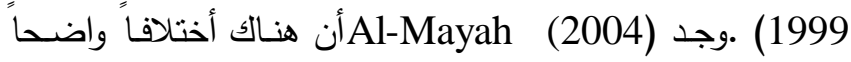
داد ي أع

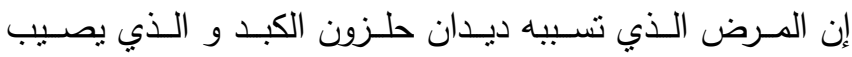

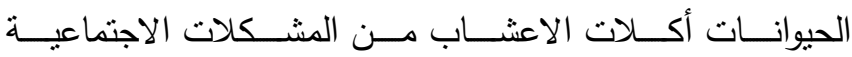

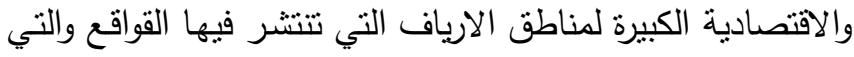

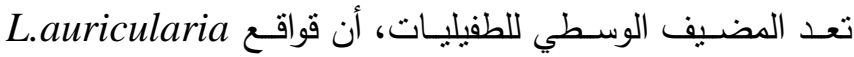
المضيف الوسطي لطفيلي F.gigantica التي تعد من أشـهر

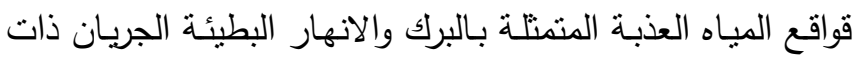

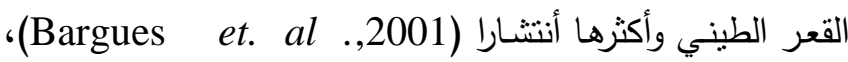

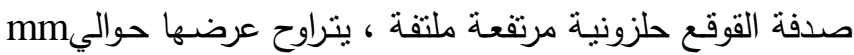
1.2-2.8 (Dundee,1974 ) ، وهـي ذات لـون بنـي مصـفر

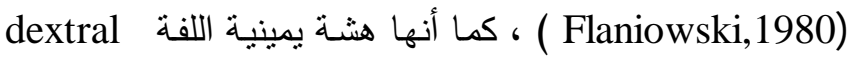

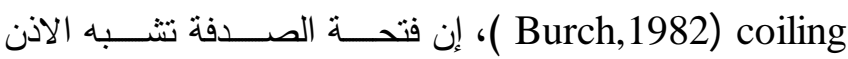
لاتمتلك غطاء ( Peckarsky et. al 1993 ; Jokinen) 1992, ; Mackie et al ., 1980

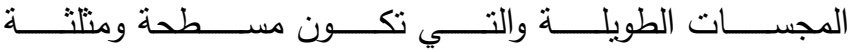
Jackiewicz,M \& R.Buksalewicz 1998 ; الثكل) (Brown , 1980 ) ، وهناك أنواع أخرى من القواقع والتي تعد

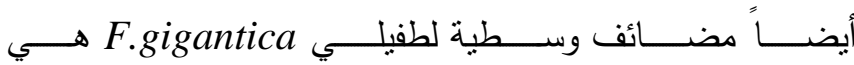
L.rubiginos و L. L. acuminatescens

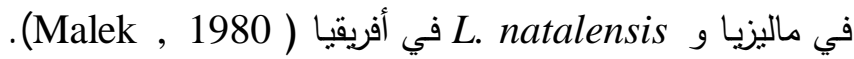
تختلف شدة الاصابة ونسبتها في المضائف الوسطبة من منطقة الى أخرى بأختلاف الظروف البيئية والاجتماعية للمنطقة إذأشار

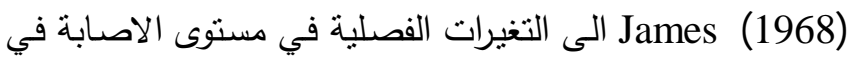

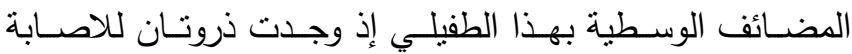
العاليةHigh Infection تحدث واحدة في أواخر الربيع (ثـهر

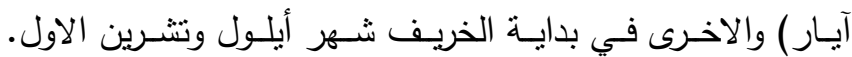

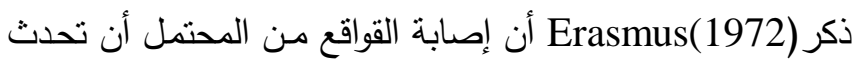

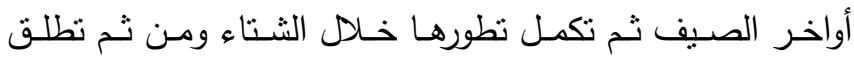

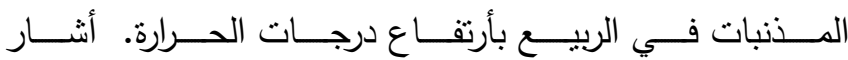
Mekroud et al al (2004) إلى إن نسبة الاصابة للقواقع بيرقـات Figantica gig في الجزائـر بلغـت 0 \% ، 2.6 \% ,

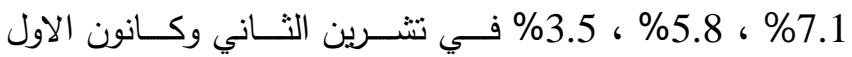
وكانون الثاني وشباط وآذار ونيسان وآيار على التوالي ـ أما في 
منطقة لرعي الحيوانات وتربيتها. تم جمع7020 عينـة من القواقع L.auricularia

F.gigantica

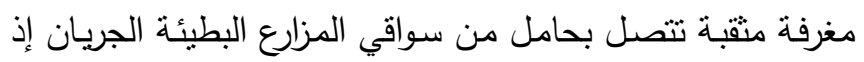
وضعت العينات في قناني بلاستيكية مفتوحة معلمة بإسم مكان

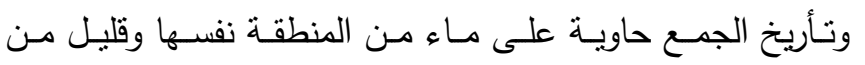

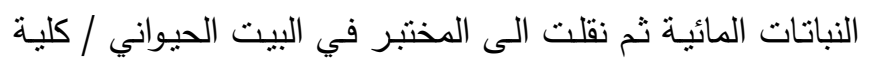

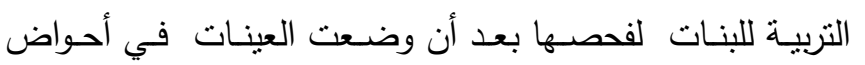

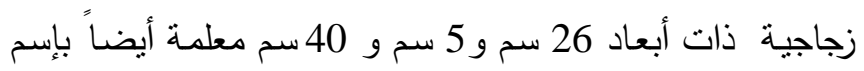

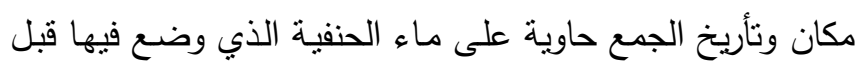

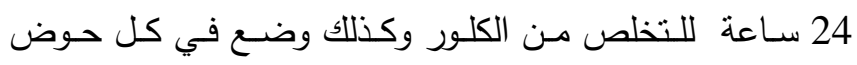
جهاز تهوية وقليل من النباتات المائية مثل الثمبلان والكرفس وتم العمل بهذه الطريقة من الجمع من الفترة ( تموز 2009 - حزيران

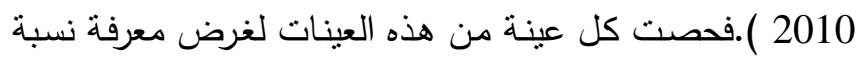
الاصابة بيرقات طفيلي F.gigantica وذلك بكسر صدفة القوقع معند بواسطة ملقط في طبق بتري يحتوي على قليل من ماء الحنفية و

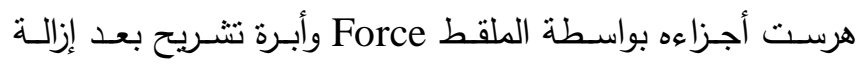

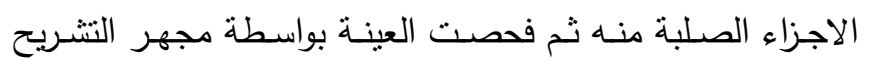
Dissecting Microscop (Thienpont et al ., 1979) (عتمد في تصنيف القواقع

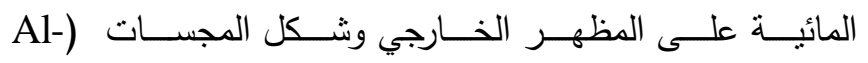
Mashhadani,1970 وتم تأكيد التشـخيص مـن قبـل الاسـتاذ

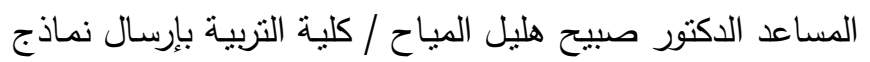
من القواقع الى جامعة البصرة.
المــنبات المنطلقـة مـن القواقـع ووجدـ أن هـذه المـنبات تقضـل

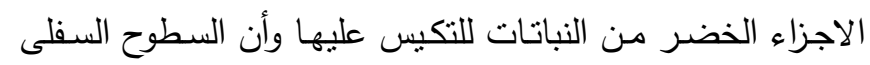

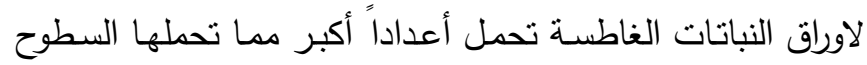

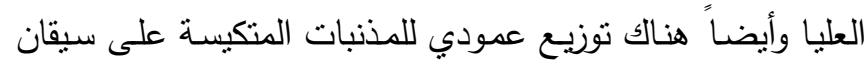
النباتـات في المـاء. هـدفت الدراســة الحاليـة الـى دراسـة وبائيـة الطفيلي في المضائف الوسطية في ثناث مناطق من المحافظة هي قضاء الكوفة والمناذرة ومنخفض بحر النجف .

\section{المو اد وطر ائق العمل: جمع وفحص عينات المضيف الوسطي} بعد إجراء مسح لمناطق متعددة في محافظة النجف الاشرف

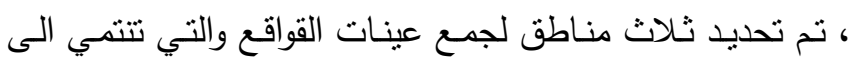
عائلـة Lymnaeidea ـ إذ كانــت المنطقـة الاولـى هـي المنـاذرة

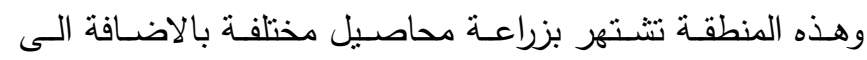

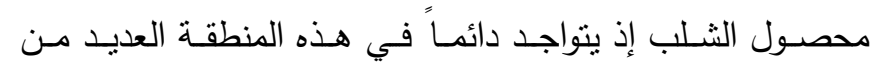

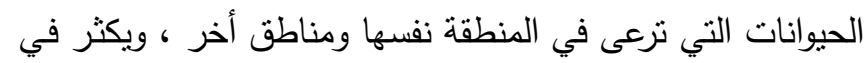

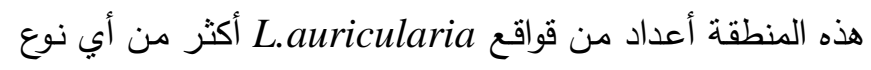
آخر مـن القواقع ويتواجد في هذه المنطقـة العديـد مـن الانهـار

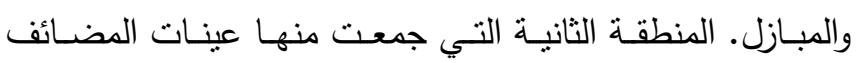
الوسطية هي بحر النجف والتي تمتاز بزراعـة محصـول الثـلب صيفاً والمحاصيل الاخرى وتكون مياه هذه المنطقة بطيئة الجريان

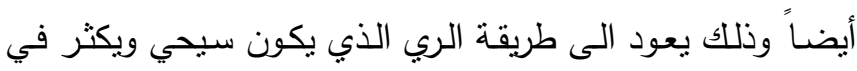
هذه المنطقة العديد من النباتات لعدم تتظيفها ـ المنطقة الثالثة

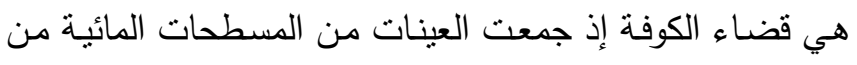

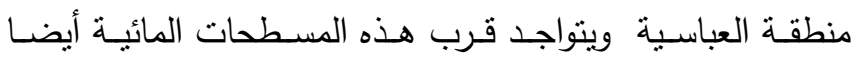


شكل بيضوي ، وأيضاً عدد لفات الحلزون التي تكون لفتين زائداً التحليل الاحصائي:

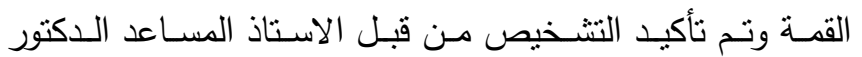

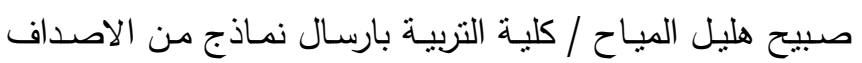

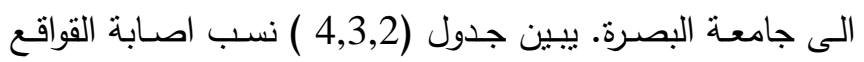
بطفيليF.gigantica في كل من قضاء الكوفة وناحية المناذرة

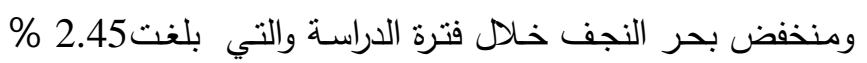
و 5.08\% و 0.92 \% على التوالي .

حللت النتائج أحصــائياً باسـتخدام جـدول تحليـل التبـاين ANOVA الدقارنات الواردة في الدراسة (الراوي وخلف ، 1980 ) ).

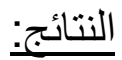

يبين الجدول (1) أن 207 قوقع مصاب من مجموع 7020 مبن

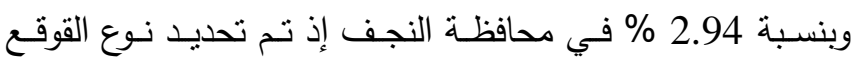
المائي L.auricularia بكون الصدفة أطول من الحلزون وذات

جدول رقم (1) نسب الاصابة بيرقات طفيلي Figantica . النجف

\begin{tabular}{|c|c|c|c|c|c|c|c|c|c|c|c|c|c|}
\hline المجموع & حزيزان & آبار & تيسان & آذار & شباط & $\begin{array}{c}2 \sqrt{3} \\
2010\end{array}$ & $1^{\text {⿹ }}$ & 2 & ت1 & أيلول & آب & تموذ 2009 & الثهر \\
\hline 7020 & 696 & 711 & 804 & 572 & 365 & 410 & 446 & 520 & 475 & 621 & 647 & 753 & الكلايد \\
\hline 207 & 0 & 0 & 39 & 3 & 26 & 40 & 46 & 34 & 11 & 0 & 5 & 3 & المسـاب \\
\hline 2.94 & 0 & 0 & 4.85 & 0.52 & 7.12 & 9.75 & 10.31 & 6.53 & 2.31 & 0 & 0.77 & 0.39 & $\begin{array}{c}\text { النئوبية\%\% } \\
\text { النسبة }\end{array}$ \\
\hline
\end{tabular}

تمـوز0.39 \% ولـم تظهـر اصـابة خـلال شـهري آيـار وحزيـران ونول.
يبين الجدول أعلاه أعلى نسبة اصـابة في محافظة النجف في شهر كانون الاول 10.31\% في حين اقل نسبة اصابة في شهر الجري

\begin{tabular}{|c|c|c|c|c|c|c|c|c|c|c|c|c|c|}
\hline المجموع & حزيزان & أيلز & نيسان & آذارن & شباط & $\begin{array}{c}2 \sqrt{3} \\
2010\end{array}$ & 15 & ت & ت1 & آيلول & آب & تموذ 2009 & الثهر \\
\hline 2281 & 210 & 271 & 233 & 180 & 140 & 170 & 165 & 145 & 105 & 201 & 211 & 250 & الحدد الكلى \\
\hline 56 & 0 & 0 & 0 & 3 & 7 & 3 & 26 & 15 & 2 & 0 & 0 & 0 & المصـاب \\
\hline 2.45 & 0 & 0 & 0 & 1.66 & 5 & 1.76 & 15.75 & 10.34 & 1.90 & 0 & 0 & 0 & المئوية\%\% \\
\hline
\end{tabular}

يبين الجدول أعلاه أعلى نسبة اصابة في قضاء الكوفة في شهر

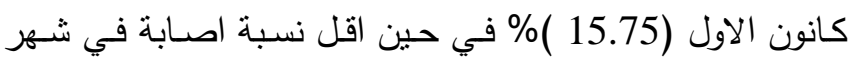
آذار (1.66)\% ولم تظهر اصابة في شهرنيسان و آيار وحزيران. 
جدول رقم (3) نسب الاصابة بيرقات طفيلي Figantica . و في المضيف الوسطي L.auricularia خلال فترة الدر اسة في ناحية المناذرة.

\begin{tabular}{|c|c|c|c|c|c|c|c|c|c|c|c|c|c|}
\hline المجموع & حزيزان & أيلز & نيسان & آذارن & شباط & $\begin{array}{c}2 \sqrt{4} \\
2010\end{array}$ & 1 ك & ت2 & ت1 & آيلول & آب & تموذ 2009 & الثهر \\
\hline 2578 & 235 & 220 & 297 & 216 & 100 & 130 & 156 & 195 & 250 & 260 & 256 & 263 & الكلتيد \\
\hline 131 & 0 & 0 & 25 & 0 & 17 & 37 & 20 & 19 & 8 & 0 & 5 & 0 & المعـاب \\
\hline 5.08 & 0 & 0 & 8.41 & 0 & 17 & 28.46 & 12.8 & 9.74 & 3.2 & 0 & 1.9 & 0 & المئوبية\%ة \\
\hline
\end{tabular}

يبين الجدول أعلاه أعلى نسبة اصابة في ناحية المناذرة في شهر آب(1.9)\% ولم تظهر اصابة في شهرأيلول و آيار وحزيران. كانون الثاني (25.46 ) \% في حين اقل نسبة اصابة في شهر

جدول رقم (4) نسب الاصابة بيرقات طفيلي Fo gigantica في المضيف الوسطي L.auricularia خلال فترة الدر اسة في منخفض بحر النجف.

\begin{tabular}{|c|c|c|c|c|c|c|c|c|c|c|c|c|c|}
\hline المجموع & حزيران & أيلز & تيسان & آذار & شباط & $\begin{array}{c}2 \sqrt{5} \\
2010\end{array}$ & 15 & ت & ت1 & أيثلول & آب & تموذ 2009 & الثهر \\
\hline 2161 & 251 & 220 & 274 & 176 & 125 & 110 & 125 & 180 & 120 & 160 & 180 & 240 & الكلى \\
\hline 20 & 0 & 0 & 14 & 0 & 2 & 0 & 0 & 0 & 1 & 0 & 0 & 3 & المعـاب \\
\hline 0.92 & 0 & 0 & 5.10 & 0 & 1.6 & 0 & 0 & 0 & 0.83 & 0 & 0 & 1.25 & المئوبية\%\% \\
\hline
\end{tabular}

فبعد نشـريح 7020 قوقـع مـائي للنوع أعـلاه، والتي جمعت مـن

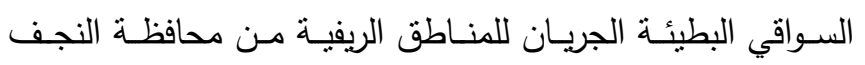
ولمـدة عـام كامـل للكثـف عـن مـدى اصـابتها بـالاطوار اليرقيـة للطفيلي F.gigantica ، وجد أن 207 منها مصـابة بـالطفيلي المذكور وبنسبة 2.94 \% جدول (1) ، وهذه النسبة لاتعد مطابقة إذا ما قورنت بنسب الاصابة المسجلة سابقاً والتي تراوحت

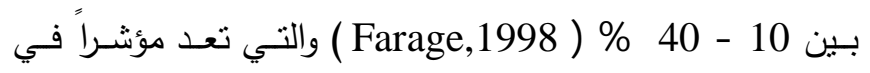
حجم التلوث العالي للمراعي والحشائش بييوض الطفيلي والناتج من انتشار الاصابة بالطفيلي بين أعداد كبيرة من الحيوانات .

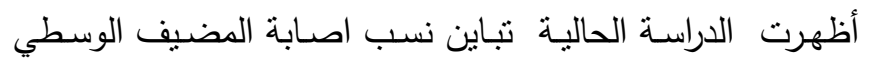
بالاطوار اليرقية لطفيلي Figantica . giاختلاف فصول السنة
يبين الجدول أعلاه أعلى نسبة اصـابة في منخفض بحر النجف

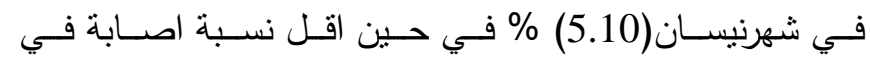
شهرتشرين الاول (0.83)\% ولم تظهر اصابة في شهرآب وأيلول

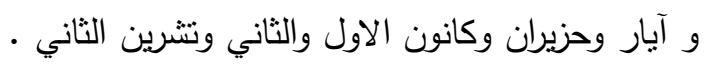

المناقشة:

إن اصابة المضيف الوسطي ( L.auricuaria ) بالطفيلي

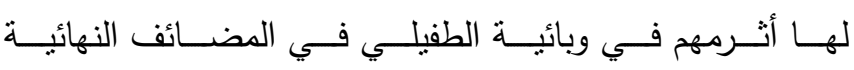

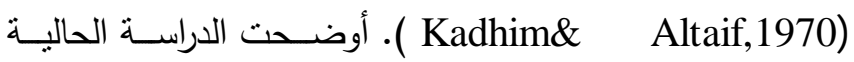

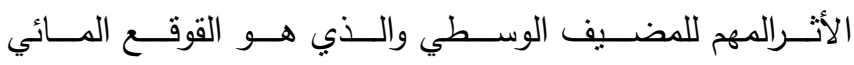
،.auricularia 
أظهرت الدراسة تركيز الاصـابة في كل من شهر تشرين

الثاني وكانون الاول وكانون الثاني وشباط ونيسان جدول (1 ) ،

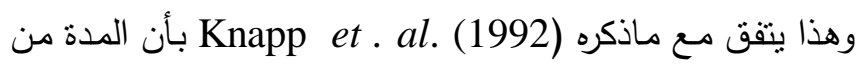

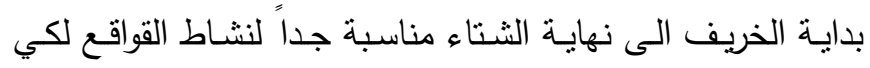

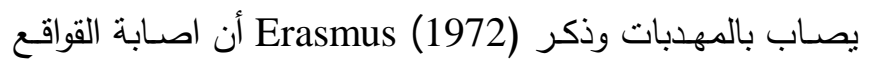
من المحتمل أن تحدث في أواخر الصيف ومن ثم تكمل تطورها

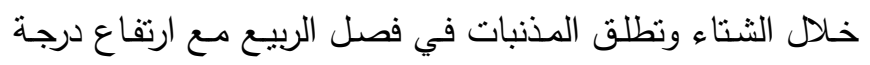

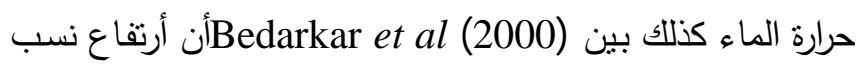
اصابة القواقع في فصل الخريف ناتج عن اصـابة القواقع باليرقات

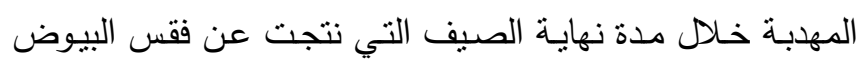

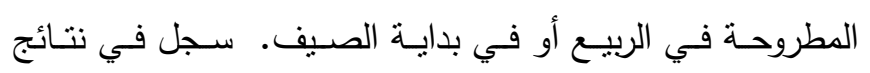

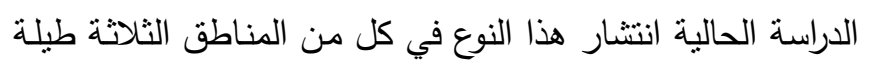

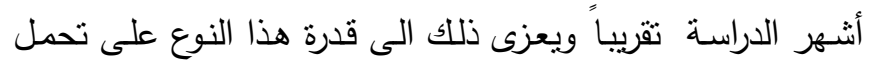

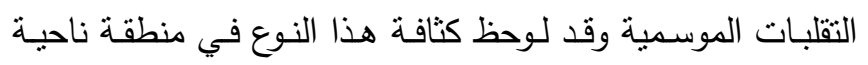
المناذرة أكثر من كثافته في المنطقتين الاخرتين وربما يعود سبب

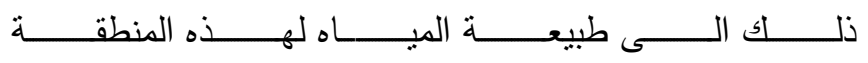

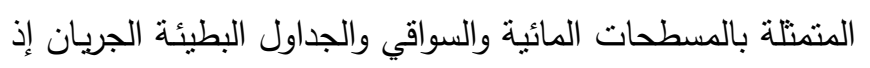

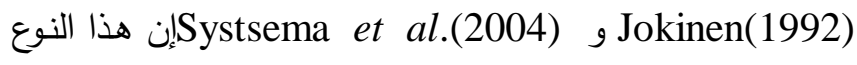
يعيش في المياه العذبة المتمتلة بالبحيرات والبرك والانهار البطيئة

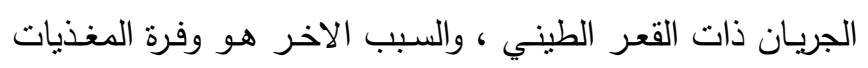

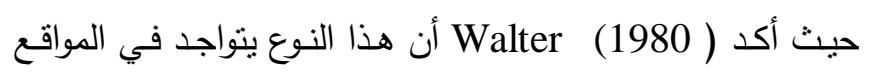
ذات المحتوى العالي من المواد الغذائية ، وقد لوحظ في الدراسـة

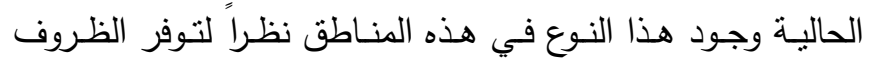

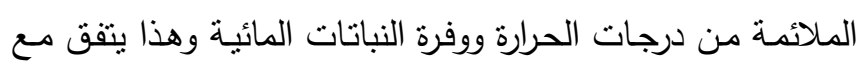
ماذكره رابع (1986 ) إذ أنثار الى نواجد أنواع أخرى من القواقع الى جنب L.auricularia مثل النوع Physa acuta والنوع تُوني . Viviparaus bengalensis
إذ سـجلت أعلى نسـبة أصـابة في الثـتاء ( 10.31\% ) و ( ) 9.75 \% ) في شـر كانون الاول كـانون الثاني على التوالي بينما أنخفضت في فصل الربيع والصيف إذ سجلت نسبة الاصـابة الى 0 \% في شـهري آيـار وحزيـران و 0.39 \% في شـهر تمـوز جدول (1). ويمكن تفسير النتائج أعلاه كون القواقع المائية غالباً ما تصـاب في فصل الخريف إذ تكون المراعي والحشـائش ملوثنة بأعداد كبيرة من بيوض الطفيلي وأن درجات الحرارة تسمح بفقس

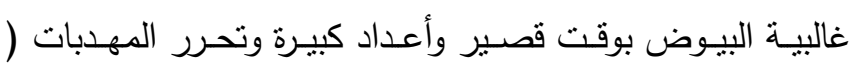

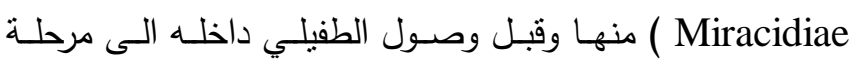

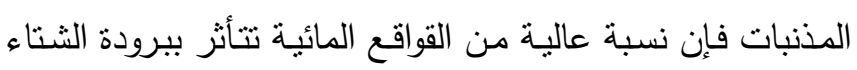

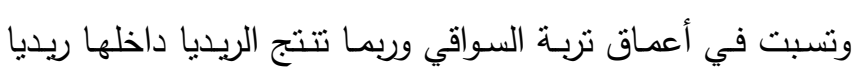
ثانوية (Daughter redia) ولهذا السبب كانت نسبة الخمج فيها عالية مع ارتفاع نسبة تواجد مرحلة الريديا ، وعند خروج القواقع المائية من سباتها في بداية الربيع تتحول الريديا بسرعة الى مرحلة

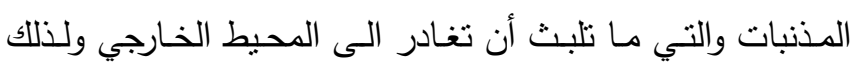
كانت نسبة الاصابة في القواقع المائية منخفضة في هذا الفصل مع انخفاض نسبة نواجد مرحلة الريديا فيها (Soulsby,1982 )

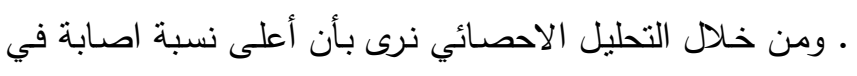
ناحية المناذرة وذلك في شـهركانون الثاني وشباط إذ بلغت نسبة نـان

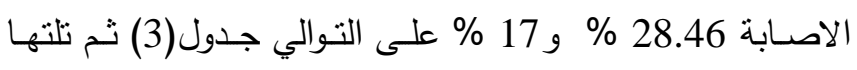

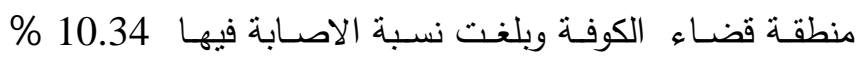
و 15.75 \% في شـهري نشرين الثاني وكانون الاول جدول (2) تلتها منطقة منخفض بحر النجف ، إذ تركزت الاصابة في شهري شـباط ونيسـان و بلغــت نسـبة الاصــابة 1.6 \% و و 5.1 \% جدول(4). عند اجراء التحليل الاحصائي تبين بأن نسب الاصابة بين منطقة ناحية المناذرة ومنخفض بحر النجف كانت معنوية وقد بـاء يعود السبب الى قرب المنطقتين و ارتبـاط ميـاه المنطقتين وقد

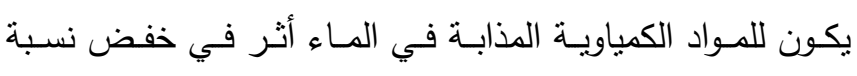
الاصابة في منخفض بحر النجف، إذ إن مياه تلك المنطقة تكون غير ملائمة لنمو وتطور الاطوار اليرقية لطفيلي F. وهذا يتفق مع ما ذكره زكريا (1979) . 
reference to Fascioliasis.M. Sc.Thesis, College of Science, Baghdad Univ.,179PP.

Al-Mayah, S .H.(2004). Studies on Serology, Biology and Control of Fasciola gigantica Cobbold,1856 infections in Basrah. Ph. D.Thesis, College of Science, Basrah Univ., 102PP.

Ashrafi, K. ; Massoud , J;Holakouei, K. ; Mahmoodi , M;Joafshani, M. A.;Valero, M.A.;Fuentes,M.V,; Khoubbane,M. ; Artigus, P. ; Bargues, M. D. and Mas- coma, S. (2004) .Evidence Suggesting that Fasciola gigantica might be the mostPrevalent causal agent of Fascioliasis in northern Iran. Iranian J. Publ. Health., 33:31-37.

Bargues , M . D. ,Vigmo, M ., Horak, P. Dvorak, J. Patzner, R . A., pointier, J .P., Jackiewicz , M ., Meier-Brook , C . , Mas-coma, S. , (2001) European, Lymnaeidae (Mollusca: Gastopoda), Intermediate hosts of trematodiases, based on nuclear ribosomal DNAITS- 2 sequences Infect Genrt Evol . 185-107.

Bedarkar, S . N.; Narladkar, B. W. and Deshpande, P. D. (2000) .Seasonal prevalence of snail born fluke infection in

Brown , D . S.(1980). Fresh water snails of Africa and their medical importance. Tylor\& Francis Ltd ., London , 127PP.

Burch, J . B . (1982) . North American freshwater snails: Identification Keys , generic synonymy , supplemental notes , glossary ,references, index . Walkerana 4: 1-365 .

Dundee , D . S . (1974). Catalog of introduced mollusks east north America (north of Mexico) . Sterkiana 55: 1-37.

Erasmus, D. A . (1972). The Biology of Trematodes .1st edn., EdwardArnold.(Puplishers), London,PP:312.

Farage, H.F. (1998). Human Fascioliasis in some countries of the Mediaterranean Region.East Mediterranean Health J., 4:156-160.

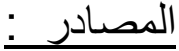

$$
\begin{aligned}
& \text { الاسـدي ، سـرمد عـواد مـوزان (2007 ). دراسـات تصـنيفية ومناعيـة } \\
& \text { حول مرض تعفن الكبد في المنطقة الجنوبية من العراق. رسالة } \\
& \text { ماجسنير ، كلية التربية ، جامعة البصرة ، } 73 \text { صفحة . }
\end{aligned}
$$

الجبوري ، مؤيـــ مجبـل عبيــ (2008 ). دراسـة وبائيـة وحيويـة حـول

أصـابة المضـائف النهائيـة والوسطية بطفيلي المتورقـة العملاقـة

Fasciola gigantica ماجسنير ، كلية النربية ، جامعة كربلاء ، 79 صفحة .

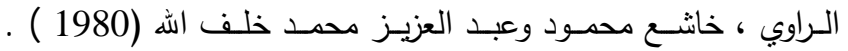

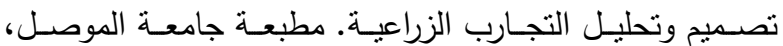
الموصل.

الصقر ،أحسان مهدي وخليل أبراهيم الطيف (1988 ) ـ علم الديدان

البيطريـة ـ مطبعـة جامعـة الموصـل ، جامعـة الموصـل ـ 368 صفحة.

الغزاوي ، غيـداء جاسـم عبـدالنبي (1995 ) • دراسـة نمـط انطـلاق

Schistosoma مــنبات طفيلـي المنشــقة الدمويــة haematobium والتأثنر العام للإصابة على المضيف الوسطي لقوقع Bulinus truncatus . رسالة ماجستنير، كليـة التربية، جامعة البصرة ، 87 صفحة .

رابع، عبد الكريم عبد الصاحب. (1986 ) .دراسة حول بيئة نوعين من

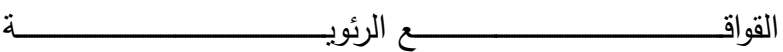

Lhysa acuta و L.auricularia ماجستير. كلية العلوم . جامعة البصرة . 131 صفحة .

زكريا ، سناء جمال (1979 ) ـ دراسات حول بعض العوامل المؤثرة

على نمو وحيوية بيوض Fasciola gigantica وفترة حياة

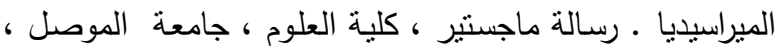
102

Al-Mashhadani, H. M. (1970). Morphology and ecology of Lymnaeid snails in Iraq with special 
Mahmoud,A-A-F.(1984). Schistosomiasis. In: Tropical and Geographical Medicine.Waren, K.S.and Mahmoud, A.A.F.(eds.) McGrow Hill Book Company, New York, PP443-360.

Malek , E . A . (1980). Snail-transmitted parasitic disease. Library of Congress cataloging in publication data, USA, 2:131-170.

Mekroud, A.; Benakhla, A.; Vignoles, P.; Rondelaud, D.and Dreyfuss, G. (2004). preliminary studies on the prevalences of natural Fascioliasis in cattle, sheep, and the host snail (Galba truncatula) in north-eastern Algeria.10. 1007/s00436 - 004- 10721.

Peckarsky, B.L., P.R. Fraissinet, M.A. Penton and D.J.Conklinjr.(1993).Freshwater Macroin vertebrates of North eastern north America .Cornell University Press, Ithaca, New York state. $442 \mathrm{pp}$.

Soulsby,E . J. L. (1982).Helminths, Arthropods and protozoa of domesticated animals. 7th. ed. Baillere, Tindall and cassell, London Pp : 809.

Systema,M .D .J .R.Cordell ,J. W . Chapman and R.C. Draheim,(2004). Lower Columbia River Aquatic no-indigenous species survey20012004.Final technical Report: Appendices prepared froThe united states coast Guard and the United States fish and Wild life services . 164PP.

Theron , A . ; Gerad, C . and Mone , H . (1992). Early enhanced Growth of the digestive of Bulibus glabrat infected with histosoma mansoni side effect or parasite manipulation .Parasitol . Res ., 78:445-450.

Thienpont,D; Rochette, F. and Vanparijs, O. (1979) Diagnosis of helminthiasis through coprological examination. Janssen Research Foundation P:26 -29 .

Thompson, S. N. (1997). Physiology and biochemistry of snail - larval trematode interaction In : Fried ,B. and Graczyk , T . K.(eds.). Advances in trematode biology. CRC press, Boca Raton, Florida , PP : 149-196.
Flaniowski , A. (1980). Pigmentation of the mantle border in polish Representatives of the subgenus Radix Lymnaeidae , (Basommatophora, Gastropoda ). Basteria 44(14):3-8.

Graczyk , T. K. and Fried , B . (1999). Development of Fasciola hepatica in the intermediate host. In: Dalton, J.P.(ed.) .Fasciolobsis. New York, 3141.

Jackiewicz, M.and R. Buksalewicz. (1998) . Diversity in tentacleShape of European lymnaeid species gastropoda monata:Basommatophora). Biological Bulletin of poznan 35(2):131-13.

James, B. L. (1968). The occurrence of larval digenea in ten species of intertidal prosobranch mollusks in Cardigan Bay . J. nat. Hist., 2:329-43.

Jokinen, E. (1992). The Freshwater Snails (Mollusca: Gastropoda) of New York State. The University of the State of New York, the State Education Department, The New York State Museum, Albany, New York 12230.112PP.

Kadhim ,J .K.and Altaif,K.I. (1970) The experimental demonstration of Lymnaea lagotis euphratica as an intermediate host of $F$. gigantica in Iraq. Ann. Trop, Med. Parasitol. 64( 3) : 335-337.

Knapp, S. E., Dunkel, A . M .; han, K and Zimmerman , L.A. (1992)Epizootiology of fascioliasis in Montana. Vet. parasitol. ,42:241-246.

Mackie, G. L., D .S . white and T. W. Zdeba. (1980). A guide to Freshwater mollusks of the Laurentian great lakes with special Emphasis on the genus pisidium . Environmental research laboratory, office of research and development, U.S.A.Environmental protection agency, Duluth, Minnesota 55804 . 144PP.

Madean,J.D. Gross , J. and Mahanty , S (1999) Liver, lung and intestinal fluke infection. In: Tropical Infectious Disease. Currant R.L.,Walker. D.H. and Willer, P.W. (eds) Academic press. USA ,PP: 1044-1050. 
level of parasitization of Lymnaea truncatula by Fasciola hepatica. J.parasitol, 76: 91-98.

Wolcott, T.G.1973.Physiological ecology and intertidal Zonation in Limpets (Acmaea): acritical look at "Limiting factors".Biological Bulletin Marine Biological Laboratory, Woods.Hole, 145:389-422.
Walter , (1980) . Density of the Pond snails Lymnaea auriculuria and Lymnaea peregra in lake Zurich Switzerland (Gastropoda: Basommatophora). Schewiz. Z. Hydrol. , 42(1):65-71.

Wilson, R. A. and Taylor, S. (1978). The effect of Variations in host and parasite density on the

\title{
Distribution of the parasite Fasciola gigantica in the intermediate host in Al-Najaf Al-Ashraf Governorate
}

\author{
Muna Hashim Gzi Jasim Hameed Rahma \\ Kufa University - Science College
}

\section{Abstract:}

This study included the detection of the Prevalence rate of Fasciola gigantica parasite in intermediate host in Najaf governorate from July 2009 to June 2010.

The intermediate host (Lymneae auricularia) collected from three regions (Bahar Al -Najaf, Kufa and Manadhera) were found to be most susceptible to be infected by this parasite.

The prevalence rate recoded in this study was $5.08 \%$ in Manadhera, $2.45 \%$ in Kufa and $0.92 \%$ in Bahar Al -Najaf. 